\title{
Dissemination of bloodmeal acquired Rickettsia felis in cat fleas, Ctenocephalides felis
}

\author{
Chutima Thepparit ${ }^{1}{ }^{*}$, Supanee Hirunkanokpun ${ }^{1}$, Vsevolod L Popov ${ }^{3}$, Lane D Foil ${ }^{4}$ and Kevin R Macaluso
}

\begin{abstract}
Background: Cat fleas, Ctenocephalides felis, are known biological vectors for Rickettsia felis. Rickettsial transmission can be vertical via transovarial transmission within a flea population, as well as horizontal between fleas through a bloodmeal. The previously undescribed infection kinetics of bloodmeal-acquired $R$. felis in cat fleas provides insight into the $R$. felis-flea interaction.
\end{abstract}

Findings: In the present study, dissemination of $R$. felis in previously uninfected cat fleas fed an $R$. felis-infected bloodmeal was investigated. At weekly intervals for 28 days, rickettsial propagation, accumulation, and dissemination in gut epithelial cells, specifically in the hindgut and the specialized cells in the neck region of midgut, were observed on paraffin sections of infected cat fleas by immunofluorescence assay (IFA) and confirmed by PCR detection of $R$. felis 17-kDa antigen gene. IFA results demonstrate ingested rickettsiae in vacuoles during early infection of the gut; lysosomal activity, indicated by lysosome marker staining of freshly-dissected gut, suggests the presence of phagolysosome-associated vacuoles. Subsequent to infection in the gut, rickettsiae spread to the hemocoel and other tissues including reproductive organs. Densely-packed rickettsiae forming mycetome-like structures were observed in the abdomen of infected male cat fleas during late infection. Ultrastructural analysis by transmission electron microscopy (TEM) confirmed the presence and infection characteristics of Rickettsia including rickettsial destruction in the phagolysosome, rickettsial division, and accumulation in the flea gut.

Conclusions: This study intimately profiles $R$. felis dissemination in cat fleas and further illuminates the mechanisms of rickettsial transmission in nature.

Keywords: Rickettsia felis, Ctenocephalides felis, Cat flea, Rickettsial infection

\section{Findings}

\section{Background}

Rickettsia felis has emerged as a cosmopolitan arthropodborne pathogen infecting various arthropod hosts. First associated with human disease in 1991, this intracellular Gram negative bacteria shares clinical signs with other rickettsial diseases, ranging from fever and generalized maculopapular rash to more severe acute polyneuropathylike symptoms [1,2]. Coinciding with the impetus to understand transmission biology, increased recognition of $R$. felis as a pathogen derives from studies revealing $R$. felis

\footnotetext{
* Correspondence: cthepparit@vetmed.lsu.edu

'Department of Pathobiological Sciences, Louisiana State University, School of Veterinary Medicine, Baton Rouge, LA 70803, USA

${ }^{2}$ Center for Vaccine Development, Institute of Molecular Biosciences, Mahidol University, Nakhon Pathom 73170, Thailand

Full list of author information is available at the end of the article
}

in approximately $6 \%$ of non-malarial, febrile Senegalese and Kenyan patients hospitalized [3,4].

Rickettsial transmission by arthropod vectors can be vertical, horizontal, or for dynamic organisms such as R. felis, both. In an endosymbiont relationship, some arthropod hosts and their respective Rickettsia spp. are strictly maintained vertically between life cycle stages typically providing a benefit to the host [5]. Alternatively, Rickettsia spp. conferring a negative fitness effect require horizontal transmission for maintenance in arthropod populations. Identification of both transmission paradigms is unique to $R$. felis. For example, in non-hematophagous insect hosts, $R$. felis infection is associated with parthenogenesis and maintained $100 \%$ transovarially. Clearance of the organism from adult Liposcelis, common booklice, results in decreased longevity, fecundity, and non-viable egg production [6,7]. Conversely, in the cat flea, Ctenocephalides felis, vertical transmission of $R$. felis varies, not influencing flea fitness, and horizontal 
transmission via cofeeding is essential for rickettsial maintenance in flea populations (reviewed in [8]).

While molecular methods confirm detection of $R$. felis in a variety of hematophagous arthropods, only cat fleas are known biological vectors. A disseminated infection exists in vertically infected fleas; $R$. felis has been identified by PCR and microscopy in the midgut epithelial cells (adult and larval fleas), as well as adult flea muscle cells, fat body, tracheal matrix, ovaries, epithelial sheath of testes, and salivary glands [9-11]. Despite the known transmission potential of cat fleas for $R$. felis, the infection kinetics of horizontallyacquired $R$. felis in cat fleas remains unexamined. The ability to infect cat fleas with $R$. felis via an infectious bloodmeal [12] and show transmission of $R$. felis between fleas [13] was recently demonstrated using an artificial host system. To better understand the infection process, the objective of the current study involved monitoring rickettsial infection in naive fleas as it was acquired via an infectious bloodmeal. A combination of PCR, immunofluorescence assay (IFA), and transmission electron microscopy (TEM) techniques were employed to track novel infection over the duration of the cat flea's adult life. Through mapping the infection process in the natural host, an appreciation of the ecology of this emerging pathogen and potential points of intervention may be identified.

\section{Methods}

\section{Source of fleas and rickettsial cultivation}

Cat fleas (C. felis Bouche) purchased from Elward II (EL-Labs, Soquel, CA) were reared as previously described [12]. The Louisiana State University (LSU) strain of $R$. felis was propagated in ISE6, Ixodes scapularis-derived cells, and the $R$. felis-infected bloodmeal preparation was carried out as described [13] prior to enumeration using BacLight viability stain kit (Molecular Probes). A negative bloodmeal control was prepared from uninfected ISE6 cells in the same manner.

\section{Experimental design}

Newly emerged, sex-separated cat fleas were pre-fed with heat-inactivated, defibrinated bovine blood for $24 \mathrm{hr}$ prior to exposure to the $R$. felis-infected or uninfected bloodmeal at a dilution of $8.6 \times 10^{10}$ rickettsiae per $\mathrm{ml}$. After $24 \mathrm{hr}$ exposure, female and male fleas in each experimental group were mixed equally and maintained on defibrinated bovine blood (non heat-inactivated) with fresh blood replaced as needed for the duration of the experiment. Fleas, for the rickettsial dissemination examination, were collected at weekly intervals for 28 days after mixing the population [designated as day post-exposure (dpe)].

\section{Genomic DNA isolation and PCR amplification}

Whole fleas or individual tissues including female gut, ovary, salivary glands, and male rectal ampulla from three fleas were pooled and gDNA was extracted from the ground tissue using the DNeasy blood \& tissue kit (Qiagen) according to the manufacturer's protocol. PCR conditions for detection of the rickettsial 17-kDa antigen gene were described previously [14].

\section{Microscopy}

Flea tissues (described above) were dissected from three fleas and placed in fixative; TEM was carried out at University of Texas Medical Branch (UTMB) as described previously [15].

\section{Immunofluorescence assay}

Collected fleas were placed in embedding cassettes and fixed for $24 \mathrm{hr}$ in $10 \%$ normal formalin. Formalin-fixed specimens were processed and sectioned as previously described [15]. When indicated, flea tissues were dissected and fixed with cold acetone. Rickettsiae in the formalinfixed sections and dissected fleas tissues were detected by IFA as previously described [15].

\section{Results and discussion}

In order to examine the incidence of rickettsial infection of cat fleas fed on R. felis-infected bloodmeal, eight fleas (four male and four female) collected at 5 dpe were analyzed for the presence of rickettsial $17-\mathrm{kDa}$ antigen gene by PCR. Results indicated all cat fleas exposed to an R. felis-infected bloodmeal were rickettsial infected (Figure 1). The absence of the $17-\mathrm{kDa}$ antigen gene from either the environmental blank or the fleas fed on an uninfected bloodmeal confirmed the absence of Rickettsia in EL fleas, as reported previously [12,14]. The success of utilizing an artificial feeding system for persistent infection of $R$. felis on cat fleas was recently demonstrated by Reif et al. [12].

Dissemination of $R$. felis in the infected cat fleas was visualized by IFA on the paraffin-embedded cat flea sections revealing that $R$. felis spread uniformly in both female and male cat fleas over 28 dpe (Figure 2). During early $R$. felis bloodmeal acquisition, rickettsiae densely accumulated in vacuoles in flea gut lumen (Figure 2, 1 dpe; Figure 3A, B).

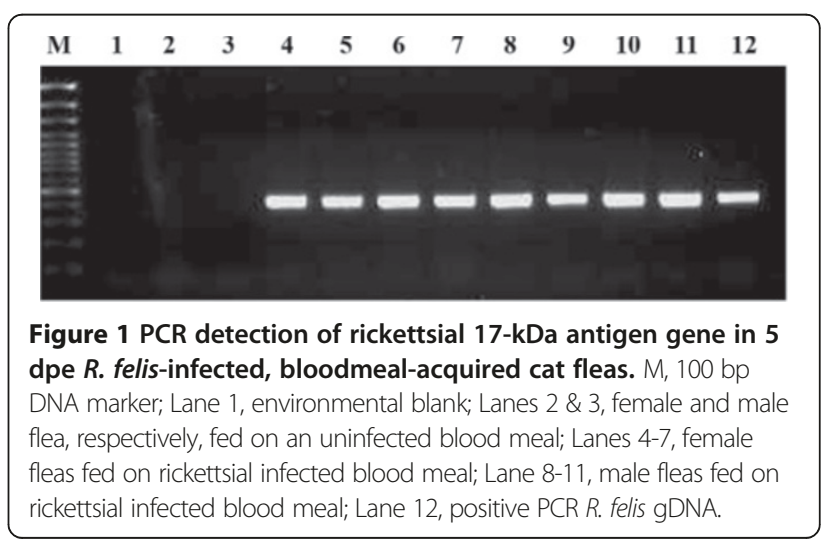


The strong signal of lysosome marker in freshly dissected $R$. felis-infected flea gut indicated that rickettsiae were associated with the phagolysosome (Figure $3 \mathrm{C}, \mathrm{D}$ ); the appearance of rickettsial lysis in a vacuole was confirmed by TEM analysis. Similar to findings in the cat flea salivary glands [10], irregular-shaped rickettsiae located in double, membrane-bound vacuoles, characteristic of rickettsiae being destroyed in a phagolysosome, were observed in $R$. felis-infected flea guts and ovaries at 28 dpe (Figure 4B, E). Ultrastructural evidence of Rickettsia-phagolysosomal lysis was previously reported in other Rickettsia-host relationships $[16,17]$. After degradation of excessive rickettsiae, remaining rickettsiae entered gut epithelium propagating primarily in the hindgut and the specialized cells forming the cardia of midgut (foregut/midgut junction) by 7 dpe (Figure 2). Within 7-14 dpe, rickettsiae spread to the entire midgut lining after which they were detected in gut lumen and occasionally found in hindgut adnexa. Between 14-21 dpe, rickettsiae migrated to the body cavity and other organs including the excretory system. The presence of Rickettsia in specific organs including gut, proventriculus, salivary glands, ovaries, and rectal ampulla was confirmed by TEM (Figure 4), IFA on dissected tissue (Figures 5 and 6 ), and PCR detection of the rickettsial $17-\mathrm{kDa}$ antigen





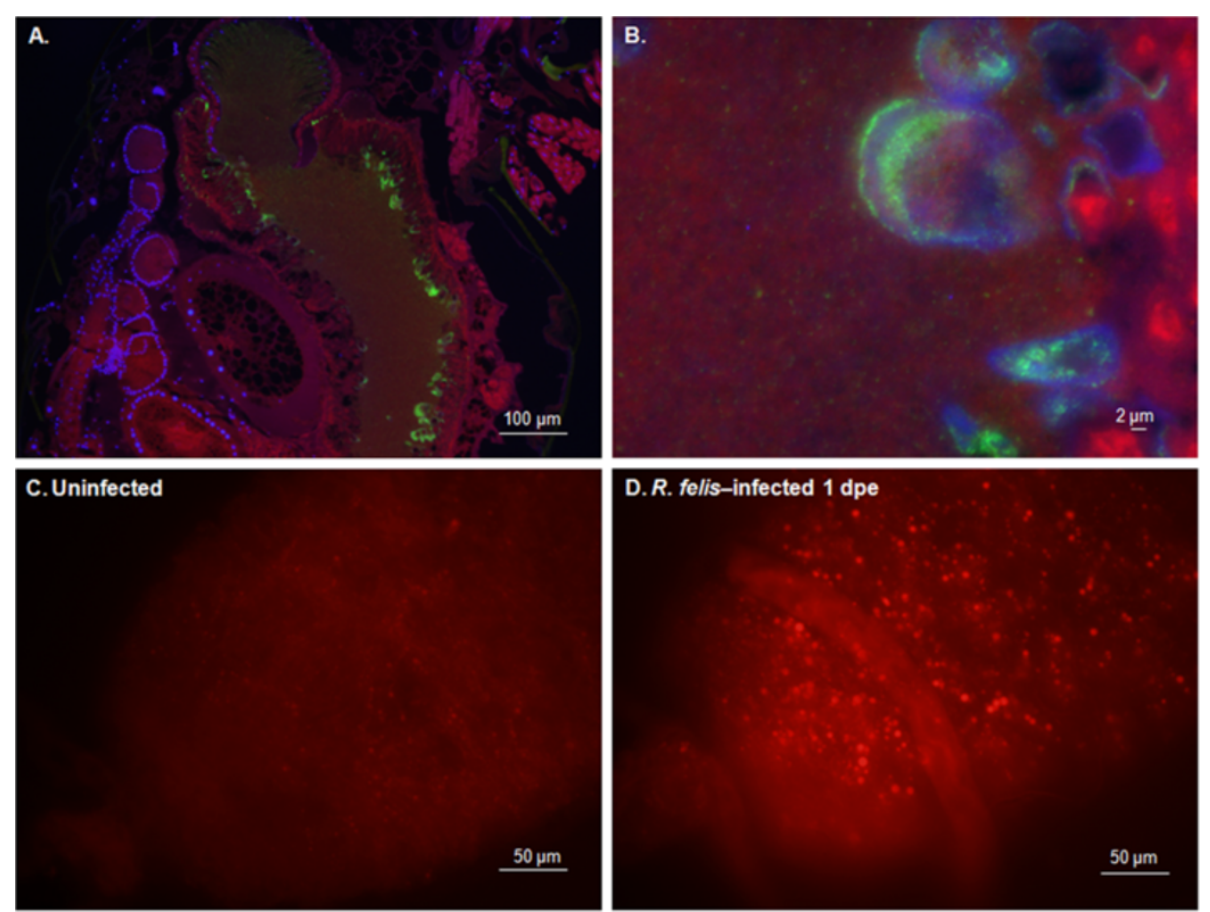

Figure 3 Rickettsia associated with phagolysosome vacuoles. A, B: Rickettsiae associated with vacuoles and freely scattered rickettsiae in gut lumen during early 1 dpe. C, D: Staining of freshly dissected guts with lysosome marker (LysoTracker ${ }^{\text {TM}}$ ) shows lysosomal activity in R. felis-infected flea gut suggesting the presence of phagolysosome.

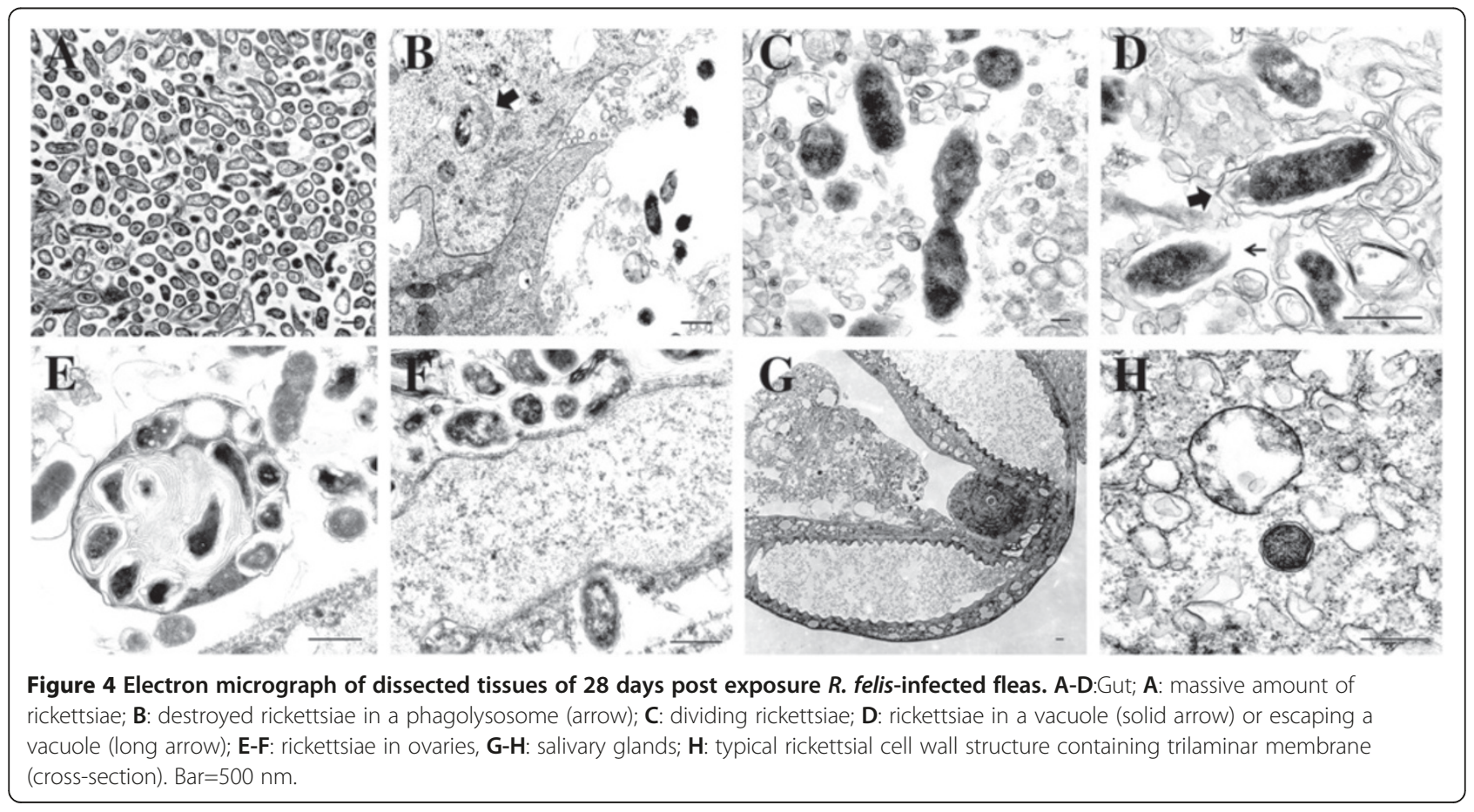




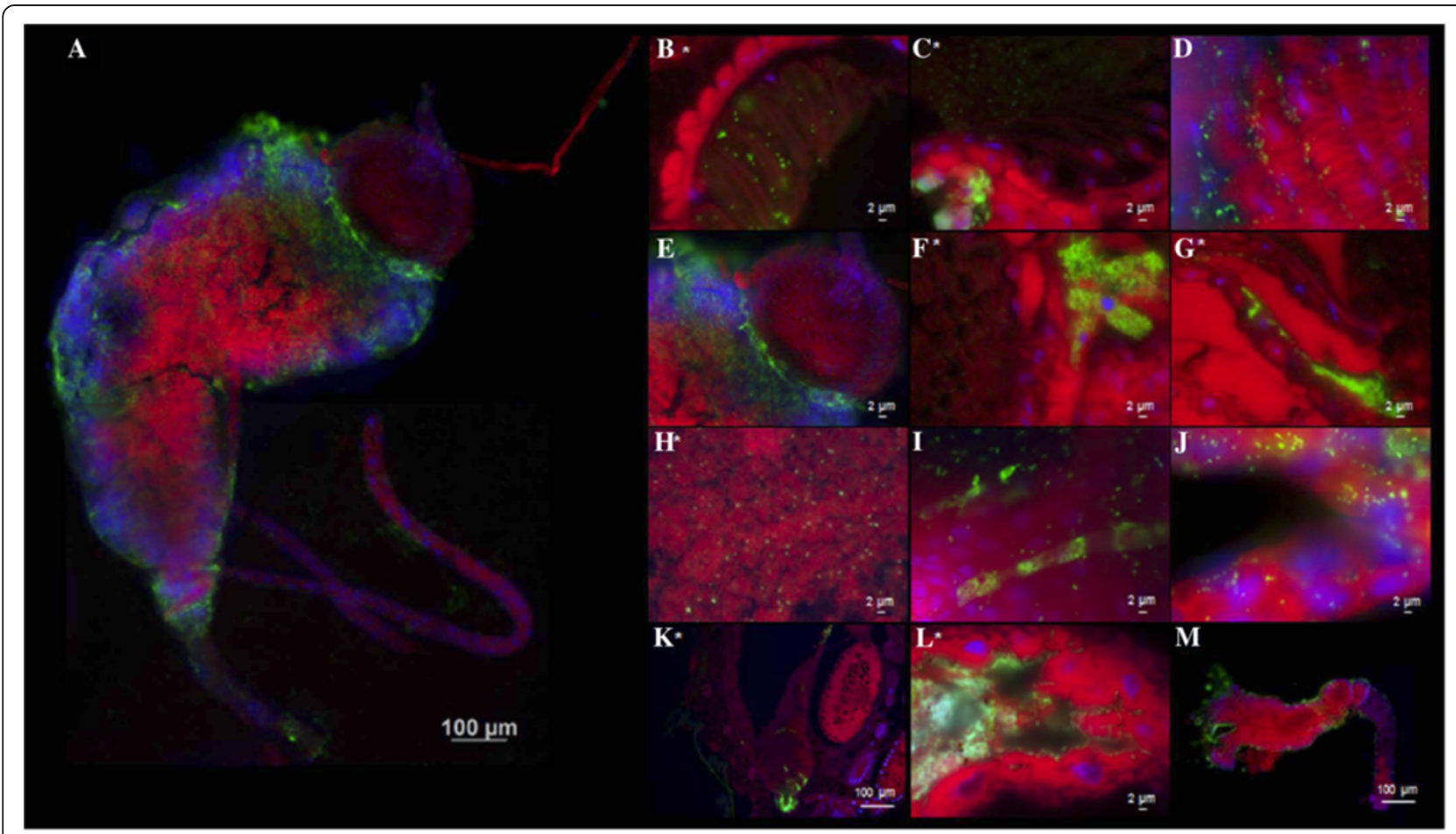

Figure 5 Dissemination of $\boldsymbol{R}$. felis in flea gut (7-14 days post-exposure). A: whole dissected gut; B-D: proventriculus; E-G: specialized cells forming the cardia (foregut/midgut junction); $\mathbf{H}$ : gut lumen; I: connective tissues on midgut (non-permeabilized-dissected gut); J: Malpighian tubules; K-M: hindgut. * IFA on paraffin-embedded sections, otherwise on dissected tissues.

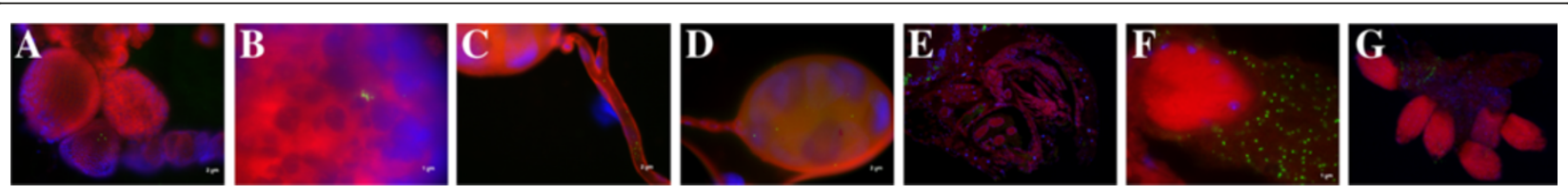

Figure 6 Dissemination of Rickettsia in flea tissues (7-14 days post-exposure). A-B: ovaries, C-D: salivary glands, E-G: rectal ampulla. * IFA on paraffin-embedded sections, otherwise on dissected tissues.

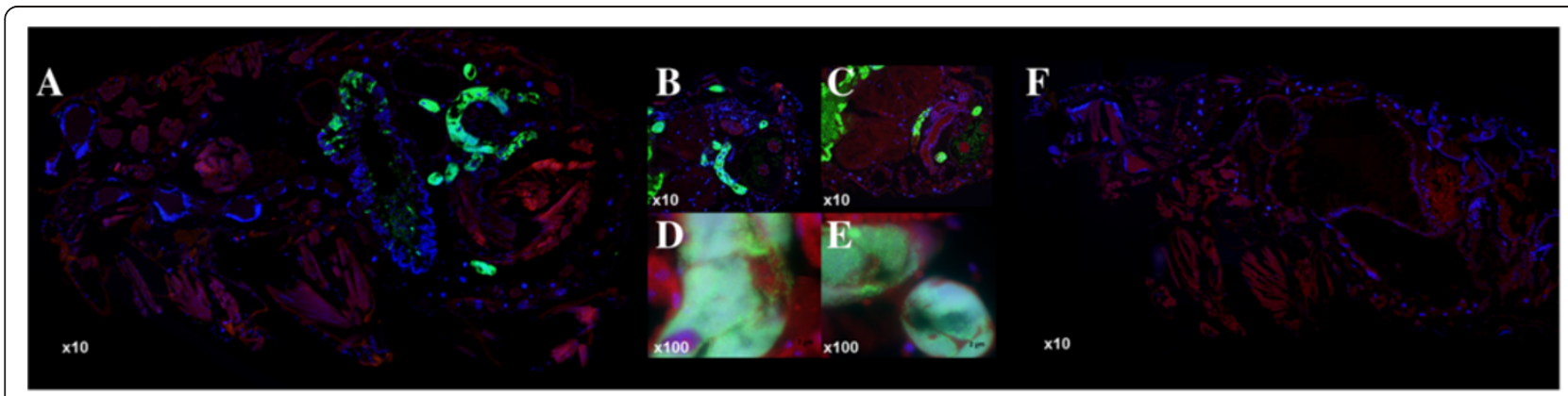

Figure 7 Rickettsiae forming mycetome-like organ located in abdomen of 28 days post-exposure $R$. felis-infected male cat fleas. A-E: R. felis-infected fleas, $\mathbf{F}:$ R. felis-uninfected flea control. 
gene (data not shown). Rickettsiae were densely aggregated around the proventriculus and its spines (Figure 5A-D). The invasion of proventriculus and midgut infection during initial infection stage was documented in tsetse fly exposed to trypanosomes via blood meal [18]. In addition to serving as a physical gut barrier, the proventriculus is thought to play a crucial role in invertebrate immunity through the expression of immune effectors and communication with other tissues to activate an adequate host immune response [19]. The evidence of clumping rickettsiae in the proventriculus and successful transmission between cat fleas suggests that Rickettsia may overcome the arthropod immune response [13].

Rickettsiae disseminated among the excretory organs including hindgut, Malpighian tubules, and rectal ampulla (Figure 1; Figure 5J-M; and Figure 6F-G) supporting rickettsiae excretion in feces, a primary mechanism for horizontal transmission $[13,20]$. Scantly detectable, rickettsiae in cat flea ovaries (Figure 4E-F; Figure 6A-B) corresponded to the variation of transovarial transmission efficiency of Rickettsia. $[8,21]$. The visualization of rickettsiae in salivary glands confirms molecular detection of $R$. felis in these organs which may be critical for horizontal transmission [10,13]. Interestingly, after 21 dpe, dense and smaller-shaped rickettsiae formed elongated clusters similar to Rickettsia-associated mycetomes in booklice [15,22]. The mycetome is primarily known to be associated with nutritional symbioses supplying essential nutrition and detoxification functions to arthropod hosts [23]. The development of single-cell mycetocytes into organ-forming mycetomes in conjunction with arthropod growth stages has been described in human head and body lice. This process is associated with the transovarial transmission of endosymbionts to the offspring [24] suggestive of the possible strategy of mycetome formation in balancing symbiont populations [25]. Rickettsia-associated mycetome-like organs previously identified in arthropods are shown to be closely associated with primary symbionts. Mycetomic rickettsiae are thought to be transitioning from pathogen and secondary symbionts to obligate reproductive or nutritional parasites and primary symbionts [22,26,27]. Although mycetomic bacteria are associated with transovarial transmission of endosymbionts, no distinct rickettsial mycetomes in female cat fleas were reported. The restriction of mycetomic rickettsiae in the $R$. felisinfected male cat fleas (Figure 7) is intriguing, requiring further elucidation.

The current study maps the rickettsial infection process of cat fleas and establishes the baseline infection kinetics towards comparison of infection by rickettsial species and between flea species. Ultimately, Rickettsiaand flea-derived factors critical to infection of fleas and subsequent transmission of $R$. felis to naïve adult cat fleas can be assessed in this system.
Competing interests

The authors declare that they have no competing interests.

\section{Authors' contributions}

$C T$ performed IFA, PCR, data analysis, and wrote the manuscript. SH carried out cat flea rearing and rickettsial infected bloodmeal acquisition. VLP conducted TEM studies. LDF and KRM participated in research design, implementation, and manuscript preparation. All authors read and approved the final manuscript.

\section{Acknowledgments}

We thank Mark Guillotte and Peter Mottram for the technical assistance and Jacqueline Macaluso for helpful comments. This research was supported by the National Institutes of Health (Al077784).

\section{Author details}

${ }^{1}$ Department of Pathobiological Sciences, Louisiana State University, School of Veterinary Medicine, Baton Rouge, LA 70803, USA. ${ }^{2}$ Center for Vaccine Development, Institute of Molecular Biosciences, Mahidol University, Nakhon Pathom 73170, Thailand. ${ }^{3}$ Department of Pathology, Center for Biodefense and Emerging Infectious Diseases, Institute for Human Infections and Immunity, The University of Texas Medical Branch, Galveston, TX 77555, USA. ${ }^{4}$ Department of Entomology, Louisiana State University, Baton Rouge, LA 70803, USA.

Received: 17 April 2013 Accepted: 21 May 2013

Published: 24 May 2013

\section{References}

1. Richter J, Fournier PE, Petridou J, Haussinger D, Raoult D: Rickettsia felis infection acquired in Europe and documented by polymerase chain reaction. Emerg Infect Dis 2002, 8:207-208.

2. Lindblom A, Severinson K, Nilsson K: Rickettsia felis infection in Sweden: report of two cases with subacute meningitis and review of the literature. Scand J Infect Dis 2010, 42:906-909.

3. Richards AL, Jiang J, Omulo S, Dare R, Abdirahman K, Ali A, Sharif SK, Feikin DR, Breiman RF, Njenga MK: Human infection with rickettsia felis, Kenya. Emerg Infect Dis 2010, 16:1081-1086.

4. Socolovschi C, Mediannikov O, Sokhna C, Tall A, Diatta G, Bassene H, Trape JF, Raoult D: Rickettsia felis-associated uneruptive fever, Senegal. Emerg Infect Dis 2010, 16:1140-1142.

5. Werren JH: Wolbachia run amok. Proc Natl Acad Sci USA 1997, 94:11154-11155.

6. Yusuf M, Turner B: Characterisation of wolbachia-like bacteria isolated from the parthenogenetic stored-product pest psocid liposcelis bostrychophila (badonnel) (psocoptera). J Stored Prod Res 2004, 40:207-225.

7. Behar A, McCormick $\amalg$, Perlman SJ: Rickettsia felis infection in a common household insect pest, liposcelis bostrychophila (psocoptera: liposcelidae). Appl Environ Microbiol 2010, 76:2280-2285.

8. Reif KE, Macaluso KR: Ecology of rickettsia felis: a review. J Med Entomol 2009, 46:723-736.

9. Bouyer DH, Stenos J, Crocquet-Valdes P, Moron CG, Popov VL, ZavalaVelazquez JE, Foil LD, Stothard DR, Azad AF, Walker DH: Rickettsia felis: molecular characterization of a new member of the spotted fever group. Int J Syst Evol Microbiol 2001, 51:339-347.

10. Macaluso KR, Pornwiroon W, Popov VL, Foil LD: Identification of rickettsia felis in the salivary glands of cat fleas. Vector Borne Zoonotic Dis 2008, 8:391-396.

11. Adams JR, Schmidtmann ET, Azad AF: Infection of colonized cat fleas, ctenocephalides felis (bouche), with a rickettsia-like microorganism. Am J Trop Med Hyg 1990, 43:400-409.

12. Reif KE, Kearney MT, Foil LD, Macaluso KR: Acquisition of rickettsia felis by cat fleas during feeding. Vector Borne Zoonotic Dis 2011, 11:963-968.

13. Hirunkanokpun S, Thepparit C, Foil LD, Macaluso KR: Horizontal transmission of rickettsia felis between cat fleas, ctenocephalides felis. Mol Ecol 2011, 20:4577-4586

14. Pornwiroon W, Pourciau SS, Foil LD, Macaluso KR: Rickettsia felis from cat fleas: isolation and culture in a tick-derived cell line. Appl Environ Microbiol 2006, 72:5589-5595. 
15. Thepparit C, Sunyakumthorn P, Guillotte ML, Popov VL, Foil LD, Macaluso KR: Isolation of a rickettsial pathogen from a non-hematophagous arthropod. PLoS One 2011, 6:e16396.

16. Kurtti TJ, Simser JA, Baldridge GD, Palmer AT, Munderloh UG: Factors influencing in vitro infectivity and growth of rickettsia peacockii (rickettsiales: rickettsiaceae), an endosymbiont of the rocky mountain wood tick, dermacentor andersoni (acari, ixodidae). J Invertebr Pathol 2005, 90:177-186

17. Walker DH, Popov VL, Crocquet-Valdes PA, Welsh CJ, Feng HM: Cytokineinduced, nitric oxide-dependent, intracellular antirickettsial activity of mouse endothelial cells. Lab Invest 1997, 76:129-138.

18. Peacock L, Cook S, Ferris V, Bailey M, Gibson W: The life cycle of Trypanosoma (nannomonas) congolense in the tsetse fly. Parasit Vectors 2012, 5:109.

19. Hao Z, Kasumba I, Aksoy S: Proventriculus (cardia) plays a crucial role in immunity in tsetse fly (diptera: glossinidiae). Insect Biochem Mol Biol 2003, 33:1155-1164.

20. Azad AF: Epidemiology of murine typhus. Annu Rev Entomol 1990, 35:553-569.

21. Higgins JA, Sacci JB Jr, Schriefer ME, Endris RG, Azad AF: Molecular identification of rickettsia-like microorganisms associated with colonized cat fleas (ctenocephalides felis). Insect Mol Biol 1994, 3:27-33.

22. Perotti MA, Clarke HK, Turner BD, Braig HR: Rickettsia as obligate and mycetomic bacteria. FASEB J 2006, 20:2372-2374.

23. Grunwald S, Pilhofer M, Holl W: Microbial associations in gut systems of wood- and bark-inhabiting longhorned beetles [coleoptera: cerambycidae]. Syst Appl Microbiol 2010, 33:25-34.

24. Perotti MA, Allen JM, Reed DL, Braig HR: Host-symbiont interactions of the primary endosymbiont of human head and body lice. FASEB J 2007, 21:1058-1066.

25. Chang KP, Musgrave AJ: Morphology, histochemistry, and ultrastructure of mycetome and its rickettsial symbiotes in cimex lectularius L. Can J Microbiol 1973, 19:1075-1081.

26. Gottlieb Y, Ghanim M, Gueguen G, Kontsedalov S, Vavre F, Fleury F, Zchori-Fein E: Inherited intracellular ecosystem: symbiotic bacteria share bacteriocytes in whiteflies. FASEB J 2008, 22:2591-2599.

27. Sakurai M, Koga R, Tsuchida T, Meng XY, Fukatsu T: Rickettsia symbiont in the pea aphid acyrthosiphon pisum: novel cellular tropism, effect on host fitness, and interaction with the essential symbiont buchnera. Appl Environ Microbiol 2005, 71:4069-4075.

doi:10.1186/1756-3305-6-149

Cite this article as: Thepparit et al: Dissemination of bloodmeal acquired Rickettsia felis in cat fleas, Ctenocephalides felis. Parasites \& Vectors 2013 6:149

\section{Submit your next manuscript to BioMed Central and take full advantage of:}

- Convenient online submission

- Thorough peer review

- No space constraints or color figure charges

- Immediate publication on acceptance

- Inclusion in PubMed, CAS, Scopus and Google Scholar

- Research which is freely available for redistribution 